Appl. Phys. A 43, 275-281 (1987)

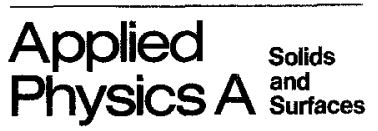

(C) Springer-Verlag 1987

\title{
Spin-Polarized Low-Energy Positron Beams and their Applications *
}

\author{
A. Rich, J. Van House, D. W. Gidley, and R. S. Conti \\ Department of Physics, The University of Michigan, Ann Arbor, MI 48109, USA \\ P. W. Zitzewitz \\ Department of Natural Sciences, The University of Michigan-Dearborn, \\ Dearborn, MI 48128, USA
}

Received 20 February 1987/Accepted 16 March 1987

\begin{abstract}
The production and use of low-energy $(100 \mathrm{eV}$ to $5 \mathrm{keV})$ high-intensity, spinpolarized positron beams is reviewed. Methods for obtaining beams with high polarization are discussed. Applications include studies of the moderation process, surface and bulk magnetism, optically active molecules, and the production of polarized anti-protons.
\end{abstract}

PACS: $34.90 .+\mathrm{q}$, 36.10.Dr, 75.30.Pd

Positrons $\left(\mathrm{e}^{+}\right)$are emitted in the decay of a radioactive nucleus with a helicity as a result of parity nonconservation in the weak interaction, permitting spinpolarized positron beams to be made easily [1]. Although the efficiency of existing moderators limits the beam intensities to a small fraction of the intensity of typical polarized electron beams, the efficiency of positron polarimeters [2] is of order 100 times larger than that of the standard electron polarimeter, so precise measurements of $\mathrm{e}^{+}$polarization can be made rapidly.

Spin-polarized low-energy positrons are useful probes of electron spins in matter as the result of spindependent interactions between electrons and $\mathrm{e}^{+}$that occur primarily in the formation of the electronpositron bound system, positronium (Ps) [3]. Groundstate Ps consists of the $1^{3} S_{1}$ (ortho-Ps or oPs) and the $1^{1} S_{0}$ (para-Ps or $\mathrm{pPs}$ ) states that can be distinguished by their different lifetimes (142 ns versus $0.12 \mathrm{~ns}$ ). The Ps atoms formed by $\mathrm{e}^{+}$and electrons with parallel spins are $100 \%$ oPs, while Ps formed from particles with anti-parallel spins is only $50 \%$ oPs. Thus pola-

\footnotetext{
* This paper is based upon an invited talk given by A.R. at the International Symposium "Production of Low-Energy Positrons with Accelerators and Applications" (Justus-Liebig-Universität Giessen, FRG, 25-27 September 1986)
}

rized $\mathrm{e}^{+}$can be used to determine the relative electron spin- state population by means of a measurement of the fraction of oPs formed. Given knowledge of the time of formation, this fraction can be determined from the lifetime spectrum. As a result Ps formation can be a sensitive probe of $\mathrm{e}^{+}$and/or electron spin-directions. In this paper we will report studies of molecular solids and ferromagnetic surfaces using polarized $\mathrm{e}^{+}$as examples of the power of this technique.

With the exception of electron capture to form Ps, the positron interactions with a solid are relatively insensitive to $\mathrm{e}^{+}$spin because the repulsion of the $\mathrm{e}^{+}$ from the atomic core reduces spin-orbit effects, and the lack of identity with electrons eliminates the Pauli exclusion principle that causes large spin-dependent effects in electron scattering. As a result, the $\mathrm{e}^{+}$spin direction is largely preserved when the $\mathrm{e}^{+}$lose energy in non-magnetic materials. Thus polarized low-energy $\mathrm{e}^{+}$beams are easily made and polarization- changing interactions are primarily the result of Ps formation events.

\section{Measuring Positron Polarization}

The measurement of $\mathrm{e}^{+}$polarization [4] is based on the mixing of the $m=0$ singlet and the $m=0$ triplet substates to form two field-perturbed states when Ps is 


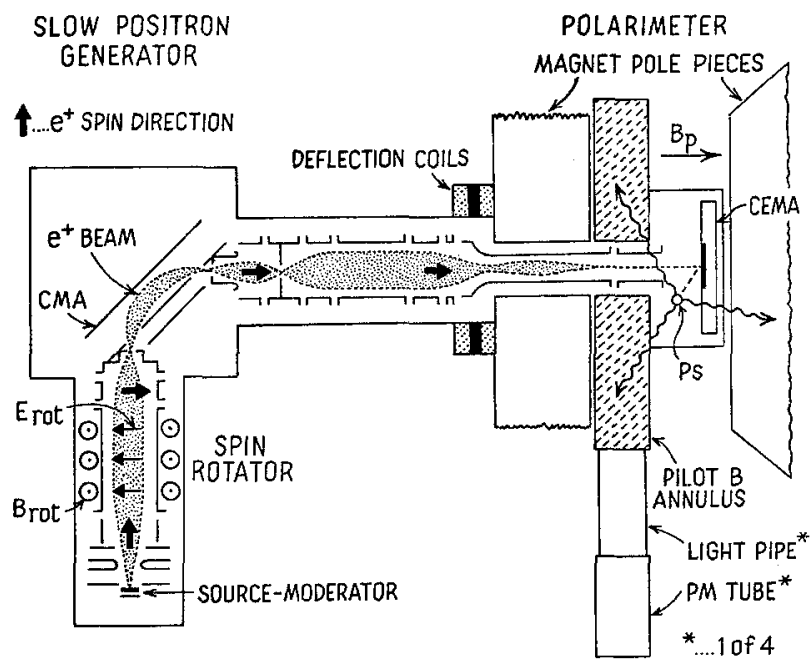

Fig. 1. Low-energy $\mathrm{e}^{+}$beam generator and polarimeter with detail of source-moderator geometry

formed in a magnetic field. The magnetic mixing not only increases the decay rate $\lambda^{\prime}$ of the perturbed triplet state, but it also makes the fraction of Ps formed in this state dependent on the quantity $\mathbf{P} \cdot \hat{B}$, where $\mathbf{P}$ is the positron polarization and $\hat{B}$ is a unit vector in the direction of the magnetic field. The decay spectrum of $o P s$ is given by

$\frac{d N(t)}{d t}=\frac{N}{4}\left[2 \lambda \mathrm{e}^{-\lambda t}+(1-\eta \mathbf{P} \cdot \widehat{B}) \lambda^{\prime} \mathrm{e}^{-\lambda^{\prime} t}\right]$.

Here $N$ is the total number of Ps atoms formed and $\lambda$ is the magnetically unperturbed $(m= \pm 1)$ decay rate including all quenching mechanisms $\left(\lambda^{-1}=142 \mathrm{~ns}\right)$. The parameter $\eta$ is given by $\eta=\chi /\left(1+\chi^{2}\right)^{1 / 2}$, where $\chi=0.276 \mathrm{~B}$ for $\mathrm{B}$ in tesla. The perturbed decay rate $\lambda^{\prime}=\left(\lambda+y^{2} \lambda_{s}\right) /\left(1+y^{2}\right)$, where $\lambda_{s}^{-1}=0.125 \mathrm{~ns}$ and $y=\chi /\left[1+\left(1+\chi^{2}\right)^{1 / 2}\right]$. At $B=0.65 T, \eta=0.176$ and $\left(\lambda^{\prime}\right)^{-1}=15 \mathrm{~ns}$. Thus decays from the perturbed triplet state can be distinguished from decays from the unperturbed state by their much higher decay rate.

The quantity $\eta P$ can be determined by measuring changes in the intensity of the perturbed triplet component relative to the intensity of the unperturbed triplet component when the $\mathrm{e}^{+}$spin direction or the applied magnetic field direction is varied. The relative intensity is measured by recording annihilation events in the background-corrected decay spectrum in two separate time windows, the perturbed window $\mathrm{W}_{1}$ (of which a fraction $f$ are composed of decays from the perturbed triplet state), and the normalization window $\mathrm{W}_{2}$ (composed $99 \%$ of unperturbed decays). The ratio $(R)$ of the number of counts in $\mathrm{W}_{1}$ to $\mathrm{W}_{2}$ is proportional to the perturbed triplet intensity. This ratio is formed first for $\mathbf{P} \cdot \hat{B}>0\left(R_{+}\right)$and then, upon reversal of the direction of either $\mathbf{P}$ or $\hat{B}$, for $\mathbf{P} \cdot \hat{B}<0\left(R_{-}\right)$. The $\mathrm{e}^{+}$ polarization is then given by

$P=\frac{1}{\eta f} \frac{\left(R_{-}-R_{+}\right)}{\left(R_{-}+R_{+}\right)}$.

Positrons emitted from the nucleus have a net helicity $h=\left\langle\boldsymbol{\sigma}_{i} \cdot \hat{p}_{i}\right\rangle=\left\langle v_{i} / c\right\rangle$. For each $\mathrm{e}^{+}, \boldsymbol{\sigma}_{i}$ is the Pauli spin matrix, $\hat{p}_{i}$ is the unit momentum, and $v_{i}$ is the speed. The ensemble of $\beta^{+}$has a net polarization $\mathbf{P}=\left\langle\boldsymbol{\sigma}_{i}\right\rangle=\left\langle\mathbf{v}_{i} / c\right\rangle$. In a beam apparatus a fraction of the emitted high energy $\beta^{+}$strikes a moderator and a small fraction of these is emitted as low-energy $\mathrm{e}^{+}$that can be collected into the beam. The polarization of the beam is strongly influenced by the selection of fast $\beta^{+}$that contribute to the $\mathrm{e}^{+}$beam.

In the Michigan polarized beam a fully electrostatic system (Fig. 1) accelerates, focuses, and transports the beam to a polarimeter. A Wien filter (crossed electric and magnetic fields) is used to rotate the $\mathrm{e}^{+}$ spin directions without disturbing the particle motion. The $\mathrm{e}^{+}$beam is projected into the polarimeter along the axis of the magnetic field through a hole in one of the pole pieces and subsequently strikes the Psformation surface. About $10 \%$ of the incident $\mathrm{e}^{+}$ capture an electron and form oPs that leaves the surface and enters the $\mathrm{MgO}$-coated confinement cavity. The polarimeter measures the lifetime of each oPs event directly by determining the formation and annihilation times. The Ps formation surface is a CEMA (chevron electron multiplier array) where the secondary electrons, generated by the impact of the $500 \mathrm{eV} \mathrm{e}^{+}$, are collected to form a pulse that indicates the time of $\mathrm{e}^{+}$arrival and Ps formation. The annihilation $\gamma$-rays are detected in plastic scintillators coupled to four photomultipliers. The time between oPs formation and annihilation is recorded either with a time-

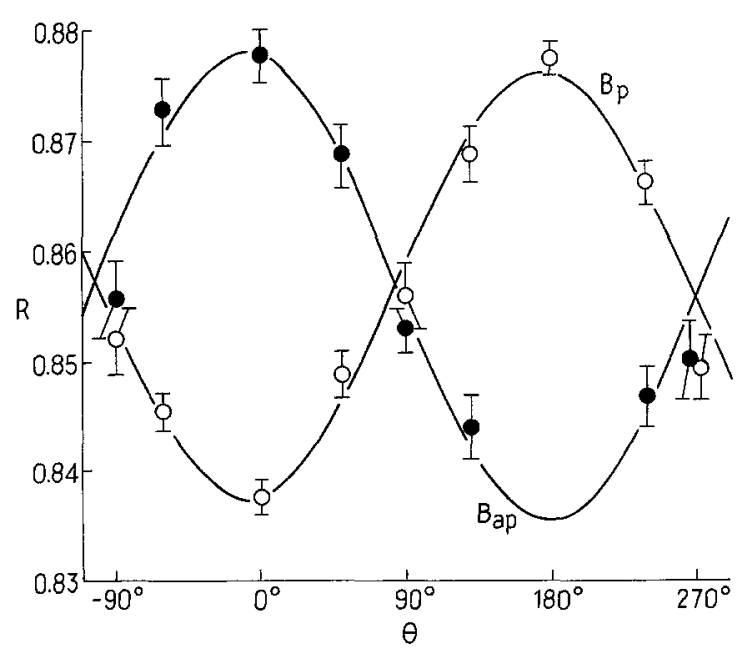

Fig. 2. Normalized perturbed oPs rate versus spin rotation angle. Curves are shown for $\mathbf{B}$ parallel and anti-parallel to beam velocity 
to-amplitude converter/multi-channel analyzer system or with a CAMAC-controlled time digitizer. The lifetime of each oPs event is thus directly measured and recorded. Operation of the polarimeter is demonstrated by the spin-rotation curves shown in Fig. 2.

\section{Producing A Highly Polarized Beam}

The polarization of the beam depends on the $\beta^{+}$ ensemble selected by the source and moderator and on changes in the directions of $\beta^{+}$spins caused by the passage of the particles through materials (including the moderator) or by stray magnetic fields. The ensemble selection includes both the angular spin distribution, determined by the angle between the emitted source $\beta^{+}$and the beam axis, and the energy distribution. Polarization is reduced by introducing into the distribution $\beta^{+}$backscattered from the source with reversed spin directions. Backscattering can be reduced by depositing the ${ }^{22} \mathrm{Na}$ on a low- $Z$ material (e.g., Be). Beam polarization is increased when the lowenergy, low-helicity $\beta^{+}$from the source spectrum are absorbed in the source material, Ti source holder window, and any additional absorbers located between source and moderator. Absorbers also increase beam polarization by preferentially absorbing those source $\beta^{+}$that are emitted at large angles relative to the axis, and thus have low polarization.

The effect of low- $Z$ (Be or plastic) absorbers on beam polarization is shown in Fig. 3. While absorbers increase polarization for the reasons discussed above, they also reduce the intensity of the $\mathrm{e}^{+}$beam. The exponential attenuation length is $(26.2 \pm 0.4) \mathrm{mg} / \mathrm{cm}^{2}$ for Be absorbers and a ${ }^{22} \mathrm{Na}$ source. Although beams with polarization as high as $P=0.66 \pm 0.03$ have been produced, a beam that optimizes $P^{2} \bar{I}$ (where $I$ is the beam intensity) has a polarization of about 0.42 and a net efficiency ( $\mathrm{e}^{+}$in beam per disintigration) $3 \times 10^{-5}$. Thus, using a $47 \mathrm{mCi}(1.7 \mathrm{GBq})^{22} \mathrm{Na}$ source, we obtain $2 \times 10^{5} \mathrm{e}^{+} / \mathrm{s}$. We note that optimization of the quantity $P^{2} I$ minimizes data collection time in a statisticslimited experiment. However, in experiments that are limited by systematic effects, it is often advantageous to maximize the signal (proportional to the polarization) even at the expense of data rate. In such a case thicker absorbers are utilized.

Depolarization can result from spin-orbit interactions that occur during various scattering processes in the absorbers and moderator. The magnitude of these processes depend on the initial energy of the incident $\beta^{+}$as well as the atomic number ( $Z$ ) of the medium [4]. Depolarization is minimized by using low-energy $\beta^{+}$ and low- $Z$ materials. In the moderator, depolarization could also occur at low energies via spin relaxation during the relatively long time required for the $\mathrm{e}^{+}$to

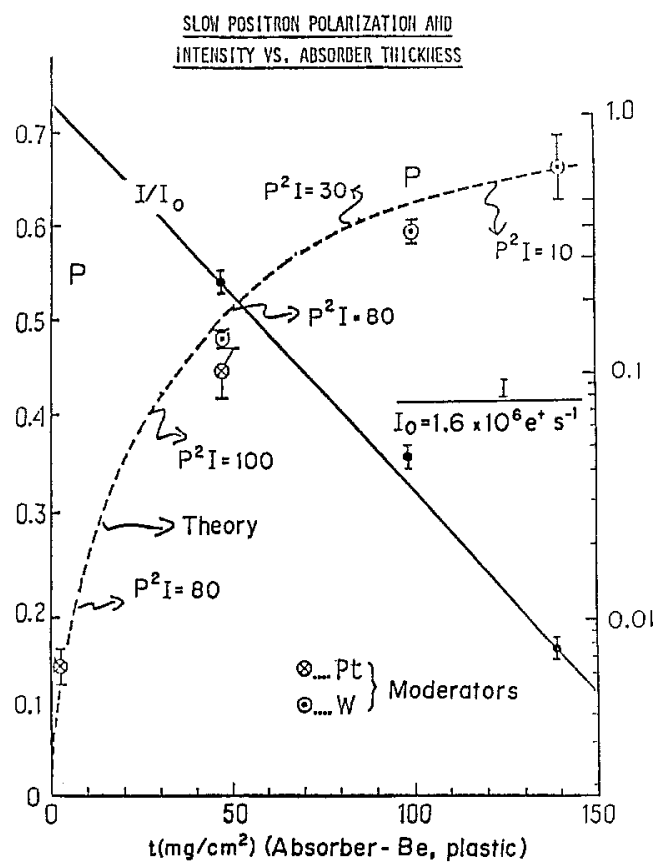

Fig. 3. Beam polarization and intensity versus absorber thickness. Dotted line shows theory [4]. Relative values of $P^{2} I$ are shown at selected values of thickness

diffuse to the surface. In ferromagnetic moderators interactions with magnetic domains can also affect polarization. Depolarization could also occur if moderation occurs via formation of Ps within or at the surface of the moderator followed by ionization to $\mathrm{e}^{+}$. Polarized beams have been used to study these processes [4].

\section{Applications of Polarized Low-Energy Positron Beams}

\subsection{Studies of the Moderation Process}

As discussed above, high-energy $\beta^{+}$are depolarized more by high- $Z$ (Pt or $\mathrm{W}$ ) moderators than by low- $Z$ ( $\mathrm{MgO}$ or Mo) moderators, while low-energy $\beta^{+}$suffer negligible depolarization in any non-ferromagnetic material. Measurements of the polarization of a beam produced by moderators of different materials under otherwise identical conditions show that the polarization is independent of the moderator $Z$ (Fig. 4). Thus, we conclude that only $\beta^{+}$incident on the moderator with very low energies are efficiently moderated. The uncertainty in the fitted slope of Fig. 4 sets an energy upper limit for significant contribution to the beam of approximately $17 \mathrm{keV}$. The role of the low $Z$ absorbers in increasing beam polarization is thus seen to be to reduce the energy of those $\beta^{+}$emitted with high polarization to energies where they can be moderated efficiently without significant depolarization [4]. 


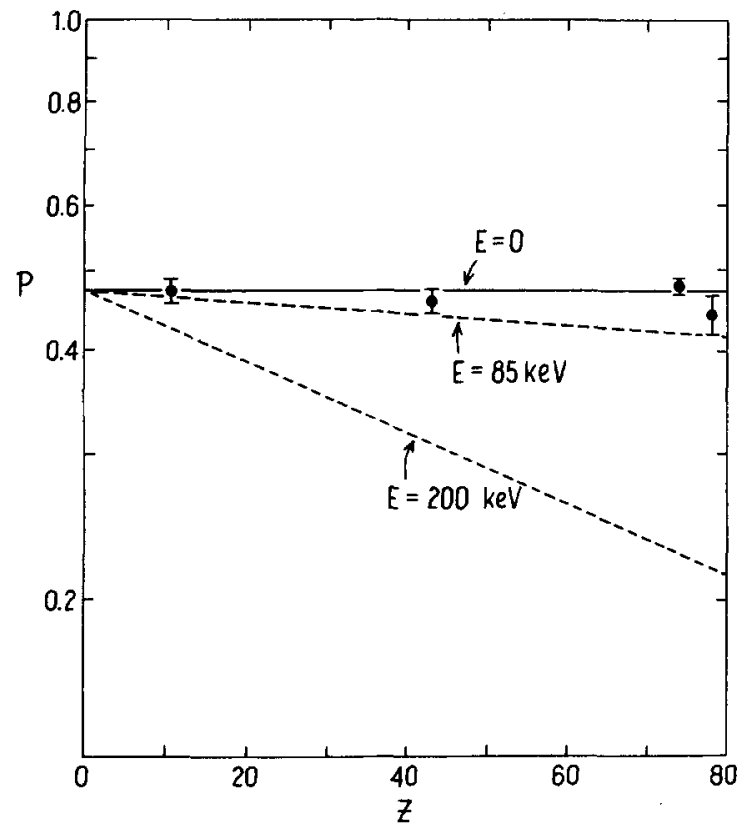

Fig. 4. Beam polarization versus moderator- $Z$. Dotted lines show theory [4] for two energies of incident $\beta^{+}$. Best fit is $E=(0 \pm 17) \mathrm{keV}$

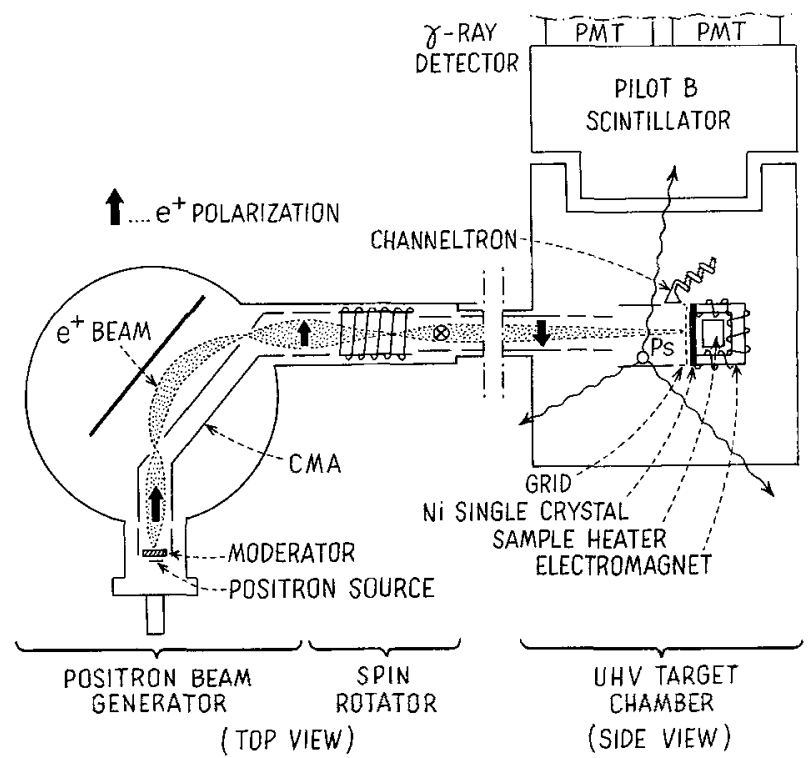

Fig. 5. Low-energy $\mathrm{e}^{+}$beam used to measure surface magnetism. Details of target chamber rotated $90^{\circ}$ for clarity

The $17 \mathrm{keV}$ upper limit is in disagreement with a current model of a moderator that assumes $\beta^{+}$implantation with an exponential profile followed by onedimensional diffusion. According to this model the average energy of $\beta^{+}$from a ${ }^{22} \mathrm{Na}$ source spectrum that contributes to the moderated beam of $64 \mathrm{keV}$, inconsistent with the upper limit derived from polarization data of $17 \mathrm{keV}$. We conclude, in agreement with the recent work of Vehanen, [6] that the true yield function has a much smaller value at high energies than the one given above.

Polarization measurements can also be used to study the emission process of $\mathrm{e}^{+}$from insulating moderators such as $\mathrm{MgO}$. Some models postulate the formation of Ps within the insulator, its escape, and subsequent ionization and release of the $\mathrm{e}^{+}$. This process would result in $\mathrm{e}^{+}$depolarization as the result of spin randomization in the $m=0$ Ps states. Depending on the lifetime of the postulated Ps, the $\mathrm{e}^{+}$ polarization would be reduced to between $67 \%$ and $50 \%$ of its initial value. However, the polarization of $\mathrm{e}^{+}$emitted from an $\mathrm{MgO}$ moderator is no less than that of $\mathrm{e}^{+}$emitted from metallic moderators, indicating that the formation of Ps does not play a significant role in the emission of $\mathrm{e}^{+}$from $\mathrm{MgO}$. Thus theories of $\mathrm{e}^{+}$emission that involve an intermediate Ps state must account for the lack of depolarization.

\subsection{Studies of Surface Magnetism}

Atoms at the surface or in a thin film of a metal have fewer neighbors than atoms in the bulk. As a result, their ferromagnetic behavior, a collective phenomenon, differs from that of atoms in the bulk. The investigation of surface and thin film magnetism tests our fundamental understanding of ferromagnetism and thus is a topic of active theoretical and experimental interest. The fact that Ps formation can occur only in the low-density tail of the electron distribution at the surface of a metal has been exploited by the Michigan-General Motors Research Laboratory group to study surface magnetism with polarized lowenergy positrons $[7,8]$.

The apparatus used to investigate surface magnetism is shown in Fig. 5. The spin-polarized $\mathrm{e}^{+}$beam is similar to the ones discussed above except that it is transversely polarized. The spin of the $\mathrm{e}^{+}$is rotated by $+90^{\circ}$ (up) or $-90^{\circ}$ (down) by an axial magnetic field before it enters the ultrahigh vacuum target chamber. The $\mathrm{e}^{+}$beam, at a selected energy between 300 and $1500 \mathrm{eV}$, is focused onto the surface of a $\mathrm{Ni}(110)$ single crystal that has been magnetically saturated parallel or antiparallel to the easy [1]1] direction (either up or down) by a current pulse through the $C$-shaped electromagnet. Lifetime techniques are used to measure an asymmetry, $A_{T}$, in the oPs formation rate,

$A_{T}=\frac{\left(R_{p}-R_{a p}\right)}{\left(R_{p}+R_{a p}\right)}$

where $R_{p}$ represents the background subtracted oPs formation rate when the sample magnetization and the $\mathrm{e}^{+}$beam polarization, $\mathbf{P}_{\mathrm{e}^{+}}$are parallel, and $R_{a p}$, is the similar formation rate when the two are anti-parallel. 


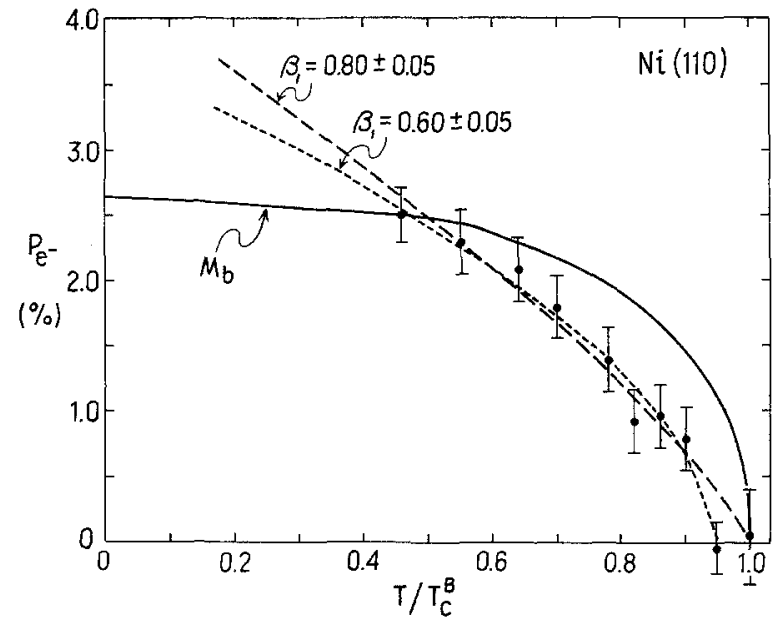

Fig. 6. Temperature dependence of $P_{\mathrm{e}^{-}}$, spin polarization of captured electrons

The polarization, $\mathbf{P}_{\mathrm{e}^{-}}$, of electrons captured at the surface to form oPs is then deduced from the relation

$A_{T}=(1 / 3)\left(\mathbf{P}_{\mathrm{e}^{+}} \cdot \mathbf{P}_{\mathrm{e}^{-}}\right)$.

The results of these initial experiments include:

1) The spin polarization of captured electrons at room temperature on $\mathrm{Ni}(110)$ is $P_{\mathrm{e}^{-}}=+(2.5 \pm 0.3) \%$ (majority spin) and changes sign on $\mathrm{Ni}(100)$ to be $P_{\mathrm{e}}$ $=-(0.9 \pm 0.1) \%$ (minority spin).

2) The size of $A_{T}$ is reduced an order of magnitude by submonolayer coverage of oxygen or Cs, as well as Ar-ion sputtering. Thus the technique is extremely sensitive to surface conditions.

3) The temperature dependence of $A_{T}$ (and thus $P_{\mathrm{e}^{-}}$) was measured in the temperature range $0.46<T / T_{c}<1.0$, where $T_{c}=633 \mathrm{~K}$ is the Ni Curie temperature (Fig. 6). Although this temperature range is too broad for a rigorous determination of critical exponents, a power law fit yields an effective exponent of $0.80 \pm 0.05$ which is in agreement with calculations as well as the results of a polarized electron scattering experiment (see references in [7]). The exponent is more than a factor of two larger than the bulk critical exponent of Ni. Thus the technique shows promise as a quantitative probe of magnetic surface critical phenomena.

\subsection{Bulk Magnetization Studies}

Information can also be obtained about the interactions of polarized low-energy $\mathrm{e}^{+}$with the electrons bound in the bulk of magnetic materials. Magnetic materials are used for constructing a moderator and the polarization of the low-energy $\mathrm{e}^{+}$emitted from the moderator surface is compared to the initial polarization of the $\beta^{+}$injected into the material. A fractional depolarization of order $\Delta P / P=\omega_{B} \tau / 2 \pi$ will occur, where $\omega_{B}=e B_{B} / m c$ is the cyclotron frequency of electrons in the bulk magnetic field $B_{B}$ and $\tau$ is the $\mathrm{e}^{+}$ thermalization and diffusion time. A measurement [4] of the polarization of low-energy $\mathrm{e}^{+}$emitted from a silicon steel moderator with randomly oriented domains shows $\triangle P / P=0.4$. Using $B_{B}=0.22 T$, we obtain $\tau=65 \mathrm{ps}$. This measurement has been confirmed by a recent measurement of the net circular polarization of the gammas resulting from $\mathrm{e}^{+}$annihilation within the bulk.

More quantitative results, allowing discrimination between diffusion and thermalization time, could be obtained by injecting a polarized $\mathrm{e}^{+}$beam into a magnetic single crystal and analyzing the phase shifts and amplitude reduction in the spin rotator curve as a function of incident $\mathrm{e}^{+}$energy.

\subsection{Interactions with Optically Active Molecules}

The net helicity imparted to positrons (and electrons) emitted in beta-decay by parity violation in the weak interaction gives them a chirality or handedness. Molecules that lack a center of inversion symmetry also have a chirality (i.e., they exist as $\mathrm{L}$ or $\mathrm{D}$ isomers). When exposed to polarized light these molecules cause a rotation of the plane of polarization, or optical activity. Many of the molecules that are the fundamental building blocks of living organisms are chiral and, in fact, exhibit a macroscopic violation of parity in that essentially only L-amino acids and D-sugars are present in living organisms.

Chiral molecules have recently [9] been predicted to have a new property, a non-zero helicity per unit volume of the outer electrons. This electron helicity density has two consequences. First, when low-energy $\mathrm{e}^{+}$with longitudinal polarization (helicity) form Ps in the $\mathrm{L}$ or $\mathrm{D}$ isomers, an asymmetry $\left(A_{\mathrm{Ps}}\right)$ is predicted to occur in the oPs formation fraction upon either reversal of the $\mathrm{e}^{+}$helicity or the exchange of the isomers. This asymmetry is predicted to be of the order of $10^{-8}<A_{\mathrm{Ps}}<10^{-6}$ for $100 \%$ longitudinally polarized, $100-200 \mathrm{eV} \mathrm{e}^{+}$incident on carbon-based targets. Second, when longitudinally polarized electrons are incident on the molecules the ionization rates are predicted to exhibit an asymmetry $A_{R}$. This asymmetry is estimated to be in the range $10^{-17}<A_{R}<10^{-11}$ for $\beta$ sources that could have been present on the early earth [10]. Thus naturally occurring betaradioactivity could have produced a small asymmetry in the ratio of $\mathrm{D} / \mathrm{L}$ isomers of the precursors of biomolecules on the primitive earth. An amplification mechanism such as an auto-catalytic reaction could have increased even the small values of $A_{R}$ given above, producing the complete asymmetry in present living 


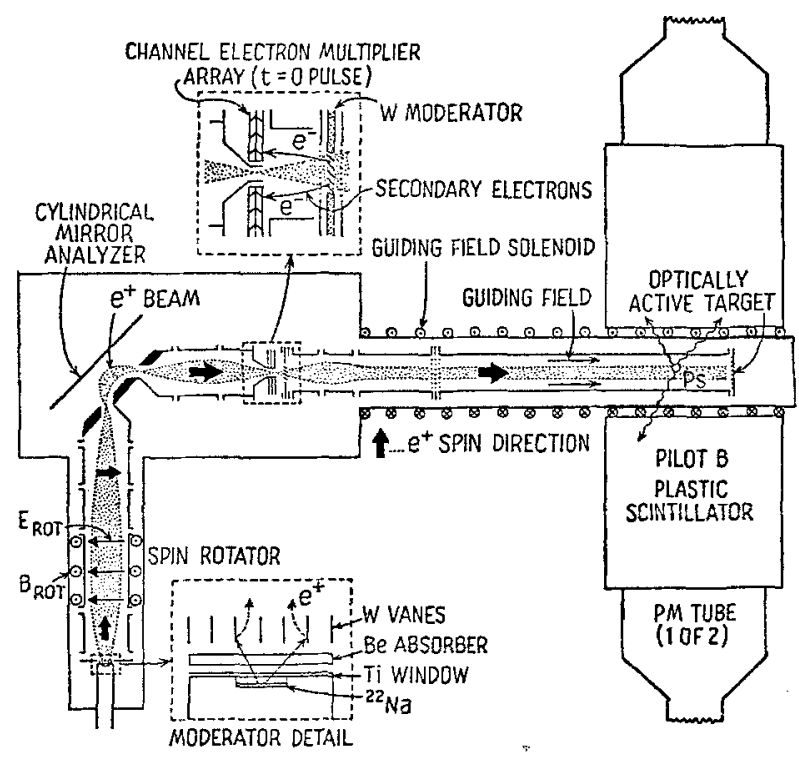

Fig. 7. Apparatus used to measure oPs formation asymmetries in optically active targets

organisms. New theoretical work [11] on autocatalysis shows that even very small biases toward one isomer could have overcome random fluctuations in the numbers of isomers so that the parity-violating weak interaction could have been the source of the macroscopic violation of parity in living organisms.

An experimental exploration of helicity density [12] uses oPs formation asymmetry $\left(A_{\mathrm{Ps}}\right)$ from lowenergy $\mathrm{e}^{+}$because it is a factor of $10^{5}$ larger than the asymmetry $\left(A_{R}\right)$ predicted for high energy betaelectrons, and because polarized $\mathrm{e}^{+}$beams and Ps lifetime techniques permit the detection of very small asymmetries. In the experimental apparatus (Fig. 7) a polarized $(P=0.48)$ low-energy $\mathrm{e}^{+}$beam is directed onto a target of amino acid powder. Calculations show that the beam helicity is partially preserved as the $\mathrm{e}^{+}$ slow down to Ps formation energies. Beam polarization is reversed every $300 \mathrm{~s}$ and $A_{\mathrm{Ps}_{\mathrm{s}}}$ is measured by determining the fraction of $\mathrm{e}^{+}$forming the long lifetime oPs. The signal for the start of the lifetime measurement is produced at the remoderator. The primary $\mathrm{e}^{+}$ beam passes through the hole in a CEMA and strikes a $\mathrm{W}$-vane remoderator at $900 \mathrm{eV}$ energy. Secondary electrons are focused back to the CEMA, providing the remoderated beam with a timing signal or "tag" that has a $50 \%$ efficiency [13]. The remoderation efficiency is typically between $10 \%$ and $20 \%$, resulting in an overall efficiency of $5 \%$ to $10 \%$. The apparatus permits measurements of asymmetries as small as $1 \times 10^{-4}$, as shown in Table 1. Theory predicts that $A_{\mathrm{Ps}} \propto Z^{2}$, prompting the use of target materials with $Z$ as high as 53 to increase the size of the asymmetry to measurable values. Note that we expect $A_{\mathrm{Ps}}\left(D D=0\right.$ and $A_{\mathrm{Ps}}(\mathrm{L})=$ $-A_{\mathrm{Ps}}(\mathrm{D})$. Part of the theoretical range is already excluded by the data in Table 1. An improved apparatus, now undergoing final tests, will have a sensitivity of $1 \times 10^{-5}$, sufficient to explore the entire theoretical range in high- $Z$ materials [14].

\section{Future Research}

Most of the applications described above were completed before the development [15] of brightnessenhanced, time-tagged beams. The lack of depolarization during remoderation means that these beams can also be designed for high polarization. With the production of ${ }^{22} \mathrm{Na}$ sources as large as $40-400 \mathrm{GBq}$ (1-10 Ci) now being developed at Michigan and Brookhaven National Laboratories, it will be possible to obtain beams with high brightness and high polarization that also have timing information obtained far from the target region. Experiments not previously possible will become practical. Some of these experiments are described below.

The same lack of spin-induced effects between $\mathrm{e}^{+}$ and atoms that allows the retention of beam polarization also makes spin-polarization effects in the collisions of $\mathrm{e}^{+}$with closed shell (rare gas) atoms vanishingly small. However, effects in atoms such as tungsten are expected to be large enough to be measured.

Scattering of low-energy electrons (LEED) and positrons (LEPD) from crystalline solids $[15,16]$ provides information on the surface of the solid. Spinpolarized electron scattering (PLEED) experiments have been shown to provide important information that can be complemented in spin-polarized lowenergy positron diffraction (PLEPD) studies. Calculations [17] on W indicate the PLEPD effects would be measurable. Remoderated, polarized $\mathrm{e}^{+}$beams now have the requisite intensity and brightness.

Additional surface magnetism studies of $P_{\mathrm{e}^{-}}$in single crystals of $\mathrm{Fe}$ and $\mathrm{Co}$ are planned. It should also

Table 1. Experimental limits for positronium formation asymmetries in chiral molecules [14]

\begin{tabular}{lcccc}
\hline Target & $Z$ & $A_{\mathrm{Ps}}(\mathrm{DL}) \times 10^{-4}$ & $A_{\mathrm{Ps}}(\mathrm{L}) \times 10^{-4}$ & $A_{\mathrm{Ps}}(\mathrm{D}) \times 10^{-4}$ \\
\hline Leucine & 6 & $-0.5 \pm 1.5$ & $-1.2 \pm 1.5$ & $-2.7 \pm 1.5$ \\
Selenocystine & 34 & $+0.1 \pm 4.1$ & $-9.0 \pm 4.6$ & $+1.4 \pm 4.6$ \\
Thyroxine & 53 & $-5.9 \pm 4.5$ & $+5.0 \pm 5.1$ & $+1.3 \pm 5.0$ \\
\hline
\end{tabular}


be possible to measure $P_{\mathrm{e}^{-}}$in certain thin $(1-5$ monolayer) magnetic films epitaxially grown on either a magnetic or non-magnetic substrate. With the implementation of brightness enhanced, time-tagged $\mathrm{e}^{+}$ beams of high intensity, the energy of oPs formed at the surface can be resolved by time-of-flight techniques [18]. This information will reveal the location in the density of states from which the captured electron originated. Understanding of the nature of Ps formation at surfaces will also be enhanced by these studies.

It has recently been proposed [19] to produce polarized antiprotons $(\overline{\mathrm{p}})$ for high-energy polarized $\overline{\mathrm{p}}$ scattering experiments by using positrons from a highpolarization, brightness-enhanced low-energy beam. In the first step anti-hydrogen atoms $(\overline{\mathrm{H}})$ will be produced using antiprotons stored in the Low Energy Antiproton Ring (LEAR) facility at CERN. A beam of polarized $\mathrm{e}^{+}$will be velocity-matched with the stored $\overline{\mathrm{p}}$ to form $\overline{\mathrm{H}}$ via the photocapture reaction, $\mathrm{e}^{+}+\overline{\mathrm{p}}$ $\rightarrow \overline{\mathrm{H}}+\gamma[18-20]$. Half of the $\mathrm{e}^{+}$polarization will be quickly transferred to the $\overline{\mathrm{p}}$ via the atomic hyperfine interaction. In fact, virtually all of the $\mathrm{e}^{+}$polarization could be transferred to the $\bar{p}$ using the Sona method (adiabatic fast passage) [22]. In either method, the $\mathrm{e}^{+}$ would then be stripped away by passing the $\overline{\mathrm{H}}$ through a thin foil, leaving the $\bar{p}$ to be reformed into a stored beam that is now polarized and could thus be useful in high-energy scattering experiments with either an unpolarized or polarized proton target, e.g. a gas jet [23]. Work is now proceeding in our laboratory on a prototype $\mathrm{e}^{+}$beam of sufficient intensity to produce a reasonable amount of $\overline{\mathrm{H}}$. The $\overline{\mathrm{H}}$ would be formed at LEAR in collaboration with a CERN, Heidelberg, Karlsruhe group under the direction of $\mathbf{H}$. Poth.

Acknowledgement. The work reported in this article is supported by the National Science Foundation under grant PHY-8403817, by the Richard Wood Company, and by The University of Michigan.

\section{References}

1. P.W. Zitzewitz, J.C. Van House, A. Rich, D.W. Gidley: Phys. Rev. Lett. 43, 1281 (1979)

2. G. Gerber, D. Newman, A. Rich, E. Sweetman: Phys. Rev. D15, 1189 (1977)

3. A. Rich: Rev. Mod. Phys. 53, 127 (1981)

4. J. Van House, P.W. Zitzewitz: Phys. Rev. A29, 96 (1984)

5. C. Bouchiat, J.M. Levy-Leblond: Nuovo Cimento 33, 193 (1964)

6. A. Vehanen, J. Mäkinen: Appl. Phys. A36, 97 (1985)

7. D.W. Gidley, A. Köymen, T.W. Capehart: Phys. Rev. Lett. 49, 1779 (1982)

8. A.R. Köymen: $\mathrm{PhD}$ Thesis, The University of Michigan (1985) (available from University Microfilms)

9. R. Hegstrom: Nature 297, 643 (1982)

10. D.K. Kondepudi, G.W. Nelson: Nature 314, 438 (1985)

11. R. Hegstrom, A. Rich, J. Van House: Nature 313, 391 (1985)

12. D.W. Gidley, A. Rich, J. Van House, P.W. Zitzewitz: Nature 297, 639 (1982)

13. J. Van House, A. Rich, P.W. Zitzewitz: Origins of Life 14, 413 (1984)

J. Van House: PhD Thesis, The University of Michigan (1982) (available from University Microfilms)

14. J. Van House, A. Rich, P.W. Zitzewitz: Origins of Life 16, 81 (1986)

15. W.E. Frieze, D.W. Gidley, K.G. Lynn: Phys. Rev. B31, 5628 (1985)

16. I.J. Rosenberg, A.H. Weiss, K.F. Canter: Phys. Rev. Lett. 44, 1139 (1980)

17. R. Feder: Solid State Commun. 34, 541 (1980)

18. K.G. Lynn, W.E. Frieze: In Positron Scattering in Gases ed. by J.W. Humberston and M.R.C. McDowell (Plenum, New York 1984) p. $165 \mathrm{ff}$.

19. R.S. Conti, A. Rich: In Low Energy Antimatter, ed. by D.B. Cline (World Scientific, Singapore 1986) p. 99

20. H. Herr, D. Möhl, A. Winnacker: In Proc. 2nd Workshop on Physics with Cooled Low Energy Antiprotons at LEAR, Erice, 1982, ed. by U. Gastaldi, R. Klapisch (Plenum, New York 1985) p. 659

21. R. Neumann, H. Poth, A. Winnacker, A. Wolf: Z. Phys. A313, 253 (1983)

22. P.G. Sona: Energia Nucleare 14, 295 (1967)

23. A. Krisch: Private communication 